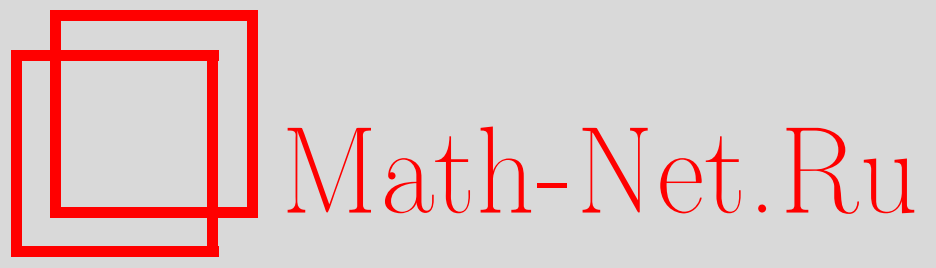

S. Albeverio, L. V. Bogachev, Localization vs. delocalization of random discrete measures, Теория вероятн. и ее примен., 1998, том 43, выпуск 4, 711734

DOI: https://doi.org/10.4213/tvp2028

Использование Общероссийского математического портала MathNet.Ru подразумевает, что вы прочитали и согласны с пользовательским соглашением

http://www . mathnet.ru/rus/agreement

Параметры загрузки:

IP : 52.6 .47 .48

26 апреля 2023 г., 11:51:40

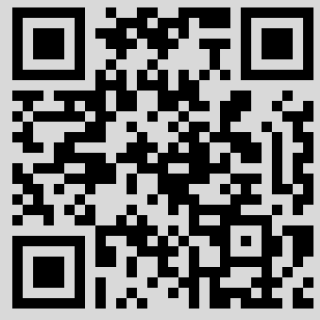


(c) $1998 \mathrm{r}$.

ALBEVERIO S. ${ }^{*}$ BOGACHEV L. V. ${ }^{* *}$

\title{
LOCALIZATION VS. DELOCALIZATION OF RANDOM DISCRETE MEASURES ${ }^{1)}$
}

\author{
Памяти Б. В. Гнеденко
}

Рассматриваются последовательности дискретных случайных мер $\mu^{(n)}$ с атомами $\left\{\mu_{i}^{(n)}, i=1,2, \ldots\right\}, \sum_{i} \mu_{i}^{(n)}=1$. Вводятся и обсуждаются понятия (полной) асимптотической локализации и делокализации таких мер в слабом (в среднем и по вероятности) и сильном (с вероятностью 1) смысле с точки зрения поведения старших атомов при $n \rightarrow \infty$. Подробно изучен класс мер с атомами вида $\mu_{i}^{(n)}=X_{i} / S_{n}$, $(i=1, \ldots, n)$, где $X_{1}, X_{2}, \ldots$ - последовательность положительных независимых одинаково распределенных случайных величин (с функцией распределения $F$ ) и $S_{n}=X_{1}+\cdots+X_{n}$. Если $\mathbf{E}\left[X_{1}\right]<\infty$, то в силу закона больших чисел для $\mu^{(n)}$ имеет место сильная делокализация. Случай $\mathbf{E}\left[X_{1}\right]=\infty$ изучен при стандартном предположении о регулярности изменения хвоста функции $F$ на бесконечности (с показателем $0 \leqslant \alpha \leqslant 1$ ). В работе показано, что при $\alpha<1$ имеет место слабая локализация. В критической точке $\alpha=1$ доказано наличие слабой дедокализации. При $\alpha=0$ локализация является сильной, если хвост распределения убывает достаточно медленно.

Ключевые слова и фразы: случайные меры, локализация, делокализация, экстремальные порядковые статистики, закон больших чисел, правильно меняющиеся функции

1. Introduction. The present paper is devoted to the asymptotic study of the sequences of random discrete measures

$$
\mu^{(n)}=\sum_{i} \mu_{i}^{(n)} \delta_{x_{i}^{(n)}}, \quad n=1,2, \ldots
$$

where $\delta_{x}$ stands for a Dirac (unit) measure concentrated at point $x$, and $\mu_{i}^{(n)}(i=1,2, \ldots)$ are positive random variables which determine the masses

*Institut für Angewandte Mathematik, Universität Bonn, D-53115 Bonn, Germany. e-mail: albeverio@uni-bonn.de

${ }^{* *}$ Faculty of Mechanics and Mathematics, Moscow State University, 119899 Moscow, Russia. e-mail: lvb@mech.math.msu.su

1) The work of the second author was partially supported by the Russian Foundation for Basic Research (project 95-01-00081) and by the Volkswagen-Stiftung (GUS-Projekt). 
of the measure atoms located at (random) points $x_{i}^{(n)}$, respectively. (In our approach, we will actually not be concerned with the structure of the set $\left\{x_{i}^{(n)}, i=1,2, \ldots\right\}$, that is, the support of $\mu^{(n)}$, and thus we identify $\mu^{(n)}$ with the aggregate $\left\{\mu_{i}^{(n)}, i=1,2, \ldots\right\}$. Such a standpoint is familiar in the probability theory where, for example, the distribution of a discrete random variable may be considered apart from the probability space on which the latter is defined. See also the remark below, in the paragraph preceding that with equation (3).) It is assumed that all the $\mu^{(n)}$ are normalized to unity:

$$
\operatorname{Var} \mu^{(n)}=\sum_{i} \mu_{i}^{(n)}=1, \quad n=1,2, \ldots
$$

The question addressed in this work is whether asymptotic localization (in the physical literature, the term «nonself-averaging» is also used [6]) of the measures $\mu^{(n)}$ takes place, that is, their discreteness is preserved (in some sense) as $n \rightarrow \infty$. Topics of that kind arise in various contexts in the theory of disordered systems where such measures may appear as probability distributions generated in random structures (see, e.g., [6], [4], [1]).

Let us point out that in general the limiting (random) measure $\mu=$ $\lim _{n \rightarrow \infty} \mu^{(n)}$ may not exist. Moreover, in many applications the support of $\mu^{(n)}$ is not specified, the atoms $\left\{\mu_{i}^{(n)}, i=1,2, \ldots\right\}$ being considered as the statistical weights of the system configurations, or «states» (cf. [4], [6]). Therefore, in order to furnish the most broad framework for the localization effects, one is led to working in the «phase space» via setting the problem purely in terms of the measure atoms $\left\{\mu_{i}^{(n)}\right\}$.

A possible way of doing this is to study certain «integral» functionals of $\mu^{(n)}$. For instance, a known device is the variation of the sequence $\mu^{(n)}$ of order $1+0$. More precisely, for $\lambda>0$ consider the variation of $\mu^{(n)}$ of order $1+\lambda$ :

$$
m_{\lambda}^{(n)}:=\sum_{i}\left(\mu_{i}^{(n)}\right)^{1+\lambda} .
$$

(Note that, according to (2), we have $\left.m_{\lambda}^{(n)}\right|_{\lambda=0}=\operatorname{Var} \mu^{(n)} \equiv 1$.) It is easy to understand that the consecutive passing to the limit as $n \rightarrow \infty$ and then $\lambda \rightarrow+0$ can help distinguish localization from delocalization. Namely, in the delocalized case $m_{\lambda}^{(n)}$ should vanish in this double limit, whereas strictly positive limit indicates the presence of a residual discrete component; moreover, approaching of $m_{\lambda}^{(n)}$ to 1 suggests that localization is «complete». This observation lies in the background of the definition of localization and delocalization in variation given below.

Another convenient approach which will be adopted in this work proceeds from the idea to study the behaviour of the senior (i.e., greatest in magnitude) of the atoms of $\mu^{(n)}$ (see [4], [13]). Indeed, it is natural to 
think that whenever complete localization occurs, the bulk mass of $\mu^{(n)}$ (say, greater than $1-\varepsilon$ with $\varepsilon>0$ arbitrarily small) stays accumulated by a few senior atoms, the number of which, as opposed to the case of delocalization, does not depend on $n$ (though it may be dependent on $\varepsilon$ ). By specifying the type of convergence of the corresponding random quantities, we will arrive to the definitions of localization and delocalization in mean, in probability and with probability 1.

The main content of the paper is devoted to studying in this framework the class of measures with the atoms of the form

$$
\mu_{i}^{(n)}=\frac{X_{i}}{S_{n}}, \quad i=1, \ldots, n,
$$

where $X_{1}, X_{2}, \ldots$ is a sequence of positive, independent, identically distributed random variables and $S_{n}=X_{1}+\cdots+X_{n}$. (The normalization condition (2) is automatically satisfied here.) Note that the random variables (4) are exchangeable (i.e., symmetrically dependent [7, section VII.4]). On account of (2), it follows that the expectation of each atom $\mu_{i}^{(n)}$ (with fixed $i$ ) is the same: $\mathbf{E}\left[\mu_{i}^{(n)}\right]=n^{-1}, \quad i=1, \ldots, n$ (cf. [4], [6]). However, this fact as it stands does not imply delocalization of $\mu^{(n)}$, but indicates that localization may take place through the accumulation of the bulk mass in a few senior atoms $\mu_{1, n} \geqslant \mu_{2, n} \geqslant \cdots$ which occur at random «places» of the sample $\left\{\mu_{1}^{(n)}, \ldots, \mu_{n}^{(n)}\right\}$.

The detailed analysis of this simple model may be instructive since (4) mimics various more realistic models which often display universal behaviour (cf. [6], [4], [13]). On the other hand, it is of interest in its own right, in particular in connection with the role played by the greatest summands in sums of independent identically distributed random variables (see, e.g., [5], [9], [2], [12], [14], [11]).

Some results on the behaviour of the measures with atoms (4) have been announced in [3]. In the present work, the complete classification of localization vs. delocalization for this model is given with full proofs.

Let us briefly outline our results. We start by discussing the general definitions of complete asymptotic localization and delocalization for the measures (1) subject to the condition (2), in the weak sense (in variation, in mean, and in probability, which prove to be equivalent to each other) and in the strong sense (with probability 1 ). We then proceed to studying the main model (4). In the simplest case where the expectation of the random variable $X_{1}$ is finite, it is easy to prove strong delocalization (Theorem 1). The case of infinite expectation is studied under the standard assumption that the upper tail of the distribution function $F(x)=\mathbf{P}\left\{X_{1} \leqslant x\right\}$ is regularly varying at infinity (with exponent $0 \leqslant \alpha \leqslant 1$ ). Theorem 3 then shows that for $0<\alpha<1$, weak localization occurs (strong localization is ruled out by Theorem 2 (ii)). The next Theorem 4 appears as a certain asymptotic version of Theorem 3. As for the critical point $\alpha=1$, Theorem 5 asserts 
that there is still delocalization; however, unlike the case $\alpha>1$ (covered by Theorem 1), for $\alpha=1$ this is only weak as indicated by Theorem 2 (i) (of course, provided the expectation is infinite). Last, in the case $\alpha=0$, where weak localization always takes place (Theorem 6), it turns out that, generally speaking, localization is strong unless the decay of the distribution tail is «hardly slow» (Theorem 7).

The paper is organized as follows. In section 2, the general definitions of various forms of weak and strong localization and delocalization are given (Definitions 1,2) and mutual relations between them are stated (Propositions 1,2). The proof of Proposition 1, being technical and quite routine, is found at the end of the paper, in Appendix (section 6). In section 3, we formulate the results (Theorems 1-7) for the model (4). A few technical lemmas, mostly well-known, are recorded in section 4 , and the proofs of the theorems are gathered in section 5 .

2. Various forms of localization and delocalization. We need to introduce some notations. Let us arrange the random atoms $\left\{\mu_{i}^{(n)}, i=\right.$ $1,2, \ldots\}$ in the decreasing order: $\mu_{1, n} \geqslant \mu_{2, n} \geqslant \cdots$. For fixed $k \geqslant 1$ we put

$$
\sigma_{k, n}:=\sum_{i=1}^{k} \mu_{i, n} .
$$

We also recall that the quantity $m_{\lambda}^{(n)}$, with $\lambda>0$, is defined in (3).

Definition 1 . We shall say that for a sequence of measures $\mu^{(n)}=\left\{\mu_{i}^{(n)}, i=1,2, \ldots\right\}$ subject to the normalization condition (2), one has (complete) asymptotic localization or delocalization:

in variation if

$$
\lim _{\lambda \rightarrow+0} \liminf _{n \rightarrow \infty} \mathbb{E}\left[m_{\lambda}^{(n)}\right]=1
$$

or, respectively,

$$
\lim _{\lambda \rightarrow+0} \limsup _{n \rightarrow \infty} \mathbf{E}\left[m_{\lambda}^{(n)}\right]=0
$$

in mean if

$$
\lim _{k \rightarrow \infty} \liminf _{n \rightarrow \infty} \mathbf{E}\left[\sigma_{k, n}\right]=1
$$

or, respectively,

$$
\lim _{k \rightarrow \infty} \limsup _{n \rightarrow \infty} \mathbb{E}\left[\sigma_{k, n}\right]=0
$$

in probability if

$$
\lim _{k \rightarrow \infty} \liminf _{n \rightarrow \infty} \mathbb{P}\left\{\sigma_{k, n}>1-\varepsilon\right\}=1, \quad \forall \varepsilon>0,
$$

or, respectively,

$$
\lim _{k \rightarrow \infty} \limsup _{n \rightarrow \infty} \mathbb{P}\left\{\sigma_{k, n}>\varepsilon\right\}=0, \quad \forall \varepsilon>0 ;
$$


with probability 1 if (in this part of the definition, all the random variables $\left\{\mu_{i}^{(n)} i, n=1,2, \ldots\right\}$ are of course assumed to be defined on a common probability space)

$$
\mathbb{P}\left\{\lim _{k \rightarrow \infty} \liminf _{n \rightarrow \infty} \sigma_{k, n}=1\right\}=1
$$

or, respectively,

$$
\mathbf{P}\left\{\lim _{k \rightarrow \infty} \limsup _{n \rightarrow \infty} \sigma_{k, n}=0\right\}=1 .
$$

Proposition 1. The conditions (L1), (L2), and (L3) are equivalent to each other and, in turn, follow from condition (L4). Similarly, (D1), (D2), and (D3) are mutually equivalent and are implied by (D4).

The proof of Proposition 1 is given in Appendix.

The conditions (D2)-(D4) can be brought to a simpler form in terms of a single, maximal atom $\mu_{1, n}$ :

$$
\begin{aligned}
\lim _{n \rightarrow \infty} \mathbf{E}\left[\mu_{1, n}\right] & =0, \\
\lim _{n \rightarrow \infty} \mathbf{P}\left\{\mu_{1, n}>\varepsilon\right\} & =0, \quad \forall \varepsilon>0, \\
\mathbf{P}\left\{\lim _{n \rightarrow \infty} \mu_{1, n}=0\right\} & =1 .
\end{aligned}
$$

Proposition 2. The conditions $\left(\mathrm{D} 2^{\prime}\right),\left(\mathrm{D} 3^{\prime}\right)$, and $\left(\mathrm{D} 4^{\prime}\right)$ are equivalent to (D2), (D3), and (D4), respectively.

This easily follows from the obvious inequalities $\mu_{1, n} \leqslant \sigma_{k, n} \leqslant k \mu_{1, n}$.

In the sequel, we will use the following terminology.

$\mathrm{D}$ e f i n it i o $\mathrm{n} 2$. In case the (equivalent) conditions (L1)-(L3) are satisfied, we shall speak of weak localization, whereas localization in the form (L4) will be called strong. Analogously, delocalization in the form (D1)-(D3) (and also $\left(\mathrm{D}^{\prime}\right),\left(\mathrm{D}^{\prime}\right)$ ) or (D4), (D4') will be referred to as weak or strong, respectively.

3. Statements of the results. We now consider the class of measures $\mu^{(n)}$ with the atoms of the form (4), where the random variables $X_{1}, X_{2}, \ldots$ are assumed positive, independent, and identically distributed (with a common distribution function $F$ ), and $S_{n}=X_{1}+\cdots+X_{n}$. Note that the decreasing-ordered atoms $\mu_{1, n} \geqslant \cdots \geqslant \mu_{n, n}$ (cf. section 2) are represented as

$$
\mu_{i, n}=\frac{X_{i, n}}{S_{n}}, \quad i=1, \ldots, n,
$$

where $X_{1, n} \geqslant \cdots \geqslant X_{n, n}$ stand for the order statistics of the sample $X_{1}, \ldots, X_{n}$.

We begin with the simplest case where the expectation of the random variables $X_{i}$ is finite (cf. [11, Lemma 4.1]). 
Theorem 1. Assume that $\mathbf{E}\left[X_{1}\right]<\infty$. Then in the model (4), the largest atom $\mu_{1, n}$ with probability 1 tends to zero as $n \rightarrow \infty$, that is, condition $\left(\mathrm{D} 4^{\prime}\right)$ is satisfied and hence strong delocalization takes place.

The case where the expectation of the random variables $X_{i}$ is infinite appears more complicated. We will study this case under the standard additional assumption of regular variation of the distribution function $F$ at infinity [7], [15].

D ef i n it i o $n$ 3. The distribution function $F(x)=\mathbf{P}\left\{X_{1} \leqslant x\right\}$ will be said to have a regular (upper) tail (with exponent $0 \leqslant \alpha \leqslant+\infty$ ) if the function $\varphi(x):=1-F(x)$ is regularly varying at infinity, that is, for every fixed $t>0$

$$
\lim _{x \rightarrow+\infty} \frac{\varphi(t x)}{\varphi(x)}=t^{-\alpha}
$$

where for $\alpha=+\infty$ the symbol $t^{-\infty}$ is defined as

$$
t^{-\infty}= \begin{cases}0, & t>1 \\ 1, & t=1 \\ \infty, & 0<t<1\end{cases}
$$

Note that if $F$ has a regular tail with the exponent $\alpha$, then $\mathbf{E}\left[X_{1}^{p}\right]<\infty$ for any $p<\alpha$ and $\mathbf{E}\left[X_{1}^{p}\right]=\infty$ for any $p>\alpha$ (see, e.g., $[10, \S 36]$ ).

Theorem 2. (i) Assume that $\mathrm{E}\left[X_{1}\right]=\infty$. Then with probability 1

$$
\limsup _{n \rightarrow \infty} \mu_{1, n}=1 \text {. }
$$

(ii) Let the distribution function $F$ have a regular tail with exponent $\alpha>0$. Then with probability 1

$$
\liminf _{n \rightarrow \infty} \mu_{1, n}=0 .
$$

This theorem amounts to saying that with probability 1 there exists a (random) subsequence of numbers $n^{\prime}$ such that $\mu^{\left(n^{\prime}\right)}$ is localized inasmuch as all the mass is eventually concentrated in a single atom (Theorem 2 (i)). On the other hand, also with probability 1 , there exists a random subsequence $n^{\prime \prime}$ such that $\mu^{\left(n^{\prime \prime}\right)}$ is delocalized, as all the atoms $\left\{\mu_{i}^{\left(n^{\prime \prime}\right)}, i=1, \ldots, n^{\prime \prime}\right\}$ are uniformly small (Theorem 2 (ii)).

$\mathrm{R} \mathrm{e} \mathrm{mark} 1$. In view of Theorem 1 , the statement (ii) of Theorem 2 is of interest only for $\alpha \leqslant 1$, otherwise $\mathbf{E}\left[X_{1}\right]<\infty$.

Theorem 3. Assume that the distribution function $F$ has a regular tail with exponent $0<\alpha<1$. Then condition (L2) is fulfilled, that is, weak localization occurs.

Note that from Theorem 2 (ii) it follows that for $0<\alpha \leqslant 1$ strong localization is ruled out.

The next theorem comes as an asymptotic version of Theorem 3 . 
Theorem 4. Let $1 \leqslant k_{n} \leqslant n$ be an arbitrary (nonrandom) sequence of integers going to $\infty$. Then under the conditions of Theorem 3 , $\lim _{n \rightarrow \infty} \mathbf{E}\left[\sigma_{k_{n}, n}\right]=1$.

The situation at the critical point $\alpha=1$ is described by the following

Theorem 5. Suppose that the distribution function $F$ has a regularly varying tail with $\alpha=1$. Then the condition (D3') holds and hence weak delocalization takes place.

It should be mentioned that if $\alpha=1$ and $\mathbf{E}\left[X_{1}\right]<\infty$, then delocalization is in fact strong (Theorem 1). It is of interest to note, however, that whenever $\mathbf{E}\left[X_{1}\right]=\infty$, this is not the case by Theorem $2(\mathrm{i})$.

Finally, consider the case $\alpha=0$ (slow variation), where one can all the more anticipate localization. In the weak version, localization immediately follows from the known results by Darling [5] and Arov and Bobrov [2] which imply that the maximal term $X_{1, n}$ completely dominates the entire sum $S_{n}$. We record this as

Theorem 6. Assume that the distribution function $F$ has a slowly varying tail, that is, (7) is satisfied with $\alpha=0$. Then the condition (L2) of weak localization holds. Moreover, $\mu_{1, n} \rightarrow 1$ in mean:

$$
\lim _{n \rightarrow \infty} \mathbf{E}\left[\left|\mu_{1, n}-1\right|^{p}\right]=0
$$

with arbitrary $p>0$, and hence in probability:

$$
\lim _{n \rightarrow \infty} \mathbf{P}\left\{\mu_{1, n}>1-\varepsilon\right\}=1, \quad \forall \varepsilon>0 .
$$

$\mathrm{R} \mathrm{e} \mathrm{m}$ a $\mathrm{rk} \mathrm{2.} \mathrm{It} \mathrm{is} \mathrm{worth} \mathrm{mentioning} \mathrm{that,} \mathrm{conversely,} \mathrm{in} \mathrm{the} \mathrm{case}$ of positive summands the slow variation proves to be as well a necessary condition for (11) (see [11, Theorem 1]).

As far as strong localization is concerned, the situation appears to be more delicate. We are able to give the complete answer to this question by adapting to our case the results by Pruitt [14] on necessary and sufficient conditions for a.s.-domination. In particular, it turns out that for $\alpha=0$ strong localization may still not take place.

Following [14], let us put

$$
v_{n}=\mathbf{P}\left\{2^{n}<X_{1} \leqslant 2^{n+1} \mid X_{1}>2^{n}\right\}=\frac{\varphi\left(2^{n}\right)-\varphi\left(2^{n+1}\right)}{\varphi\left(2^{n}\right)}
$$

where $\varphi(x)=1-F(x)$. Clearly, $v_{n}$ are fashioned so as to provide information of the distribution tail inasmuch as the slower the tail decay, the greater the $v_{n}$. Define the integer $0 \leqslant k^{*} \leqslant+\infty$ as

$$
k^{*}:=\min \left\{k \geqslant 0: \sum_{n=1}^{\infty} v_{n}^{k+1}<\infty\right\}
$$


with the convention that $\min \varnothing=+\infty$. It is easy to show that the series $\sum_{n} v_{n}$ is always divergent so that $k^{*} \geqslant 1$. Note that in the case of regular variation, from (12) and (7) it readily follows that $v_{n} \rightarrow 1-2^{-\alpha}$ and hence $k^{*} \equiv+\infty$ for $\alpha>0$. So, unlike the regularity exponent $\alpha$ which ascribes the single value $\alpha=0$ to all slowly varying functions, the critical index $k^{*}$ sets a scale for slow variation, meanwhile «sticking» all regularly varying functions (with $\alpha>0$ ) at $k^{*}=+\infty$.

Theorem 7. (i) If $k^{*}=1$ (《very slow decay») then with probability 1

$$
\lim _{n \rightarrow \infty} \mu_{1, n}=1,
$$

which implies the condition (L4) of strong localization.

(ii) If $2 \leqslant k^{*}<+\infty$ («fairly slow decay») then with probability 1

$$
\lim _{n \rightarrow \infty} \sigma_{k^{*}, n}=1,
$$

so that strong localization takes place as well. However, unlike the case (i), with probability 1

$$
\begin{aligned}
\liminf _{n \rightarrow \infty} \mu_{1, n} & =\frac{1}{k^{*}}, \\
\limsup _{n \rightarrow \infty} \mu_{1, n} & =1,
\end{aligned}
$$

and, furthermore,

$$
\begin{aligned}
\limsup _{n \rightarrow \infty} \mu_{k^{*}, n} & =\frac{1}{k^{*}}, \\
\liminf _{n \rightarrow \infty} \sigma_{k^{*}-1, n} & =1-\frac{1}{k^{*}} .
\end{aligned}
$$

(iii) If $k^{*}=+\infty$ (《hardly slow decay») then with probability 1

$$
\liminf _{n \rightarrow \infty} \mu_{1, n}=0
$$

which rules out strong localization.

$\mathrm{Re} \mathrm{m}$ a rk 3. Qualitatively speaking, this theorem indicates that with growth of the critical index $k^{*}$, localization becomes, so to say, more and more weak since the mass is essentially concentrated in exactly $k^{*}$ atoms, as evidenced by (15) and (19). In particular, in the intermediate case $2 \leqslant k^{*}<\infty$, localization cannot take place in the «amplified» form (14) because of (16). Moreover, (18) means that for any $\varepsilon>0$

$$
\mathbb{P}\left\{\mu_{1, n}>\frac{1-\varepsilon}{k^{*}}, \ldots, \mu_{k^{*}, n}>\frac{1-\varepsilon}{k^{*}} \text { i.o. }\right\}=1,
$$

(Here and below, we use the abbreviation «i.o.» for «infinitely often»; more precisely, for a sequence of events $A_{n}$, the event $\left(A_{n}\right.$ i.o.) is defined to be 
equal to $\lim \sup A_{n}=\cap_{n} \cup_{m \geqslant n} A_{m}$.) which amounts to saying that a.s. there exists a (random) subsequence of numbers $\left(n^{\prime}\right)$ such that for $\mu^{\left(n^{\prime}\right)}$ almost the entire mass is shared approximately in equal parts among the $k^{*}$ senior atoms.

$\mathrm{R} \mathrm{e} \mathrm{m}$ a r k 4. Note that in the case of regular variation, the statement (iii) implies Theorem 2 (ii) since, as mentioned above, $k^{*}=+\infty$ for all $\alpha>0$.

To conclude this section, let us show by examples that in the case $\alpha=0$ all the possibilities (i)-(iii) are realizable. In the following example (see [14]) the index $k^{*}$ can assume each of the finite values $1,2, \ldots$.

$\mathrm{Ex}$ a m p le 1 . Let $\varphi(x) \equiv \exp \left(-(\ln x)^{a}\right), x \geqslant 1$, where $0<a<1$. It is straightforward to check by the definition (7) that $\varphi$ is a slowly varying function. From (12) one easily obtains

$$
\begin{aligned}
v_{n} & =1-\exp \left(-\left(\ln 2^{n}\right)^{a}+\left(\ln 2^{n+1}\right)^{a}\right) \\
& =1-\exp \left(-(n \ln 2)^{a}\left[\left(1+\frac{1}{n}\right)^{a}-1\right]\right) \sim a(\ln 2)^{a} n^{a-1} \quad \text { as } n \rightarrow \infty,
\end{aligned}
$$

which in view of (13) implies that $k^{*}=1$ if $0<a<\frac{1}{2}$ and $k^{*}=k \geqslant 2$ whenever $(k-1) / k \leqslant a<k /(k+1)$.

Finally, the next example demonstrates that the slow variation is compatible with the possibility $k^{*}=+\infty$, that is, for $\alpha=0$ it may be the case that localization is weak rather than strong (see (20)).

$\mathrm{E} \times \mathrm{am} \mathrm{ple} \mathrm{2.} \mathrm{For} x \geqslant e$, let

$$
\varphi(x)=\exp \left(-\int_{e}^{x} \frac{d y}{y \ln \ln y}\right)=\exp \left(-\int_{1}^{\ln x} \frac{d z}{\ln z}\right) .
$$

Application of the so-called Karamata representation (e.g., [15, section 0.4, Corollary to Theorem 0.6]; see also Lemma 4 below) readily shows that $\varphi$ is slowly varying. Furthermore,

$$
v_{n}=1-\exp \left(-\int_{n \ln 2}^{(n+1) \ln 2} \frac{d z}{\ln z}\right) \sim \int_{n \ln 2}^{(n+1) \ln 2} \frac{d z}{\ln z} \sim \frac{\ln 2}{\ln n},
$$

so that $\sum_{n} v_{n}^{k}=\infty$ for all $k>0$ and hence $k^{*}=+\infty$.

4. Auxiliary lemmas. We record here a few (mostly well-known) auxiliary assertions which will be instrumental in the sequel.

Let us define the generalized inverse of the function $\varphi(x)=1-F(x)$ as (cf. [15, section 0.2])

$$
\varphi^{\leftarrow}(u):=\inf \{x \geqslant 0: \varphi(x) \leqslant u\}, \quad 0 \leqslant u \leqslant 1,
$$


with the convention that $\inf \varnothing=+\infty$. It is easily checked that $\varphi^{\leftarrow}$ is nonincreasing and right-continuous. From the definition, it also follows that

$$
\varphi^{\leftarrow}(u) \leqslant x \quad \text { if and only if } u \geqslant \varphi(x) .
$$

The following simple fact (see, e.g., [15, section 0.2]) allows one to make the so-called quantile transformation and to pass from the random variables $X_{i}$ to uniform random variables $U_{i}$ and, moreover, from the original order statistics $X_{1, n} \geqslant \cdots \geqslant X_{n, n}$, involved in (6), to the corresponding uniform order statistics, $U_{1, n} \leqslant \cdots \leqslant U_{n, n}$ (for convenience of further notation, we work with $\left\{U_{1}, \ldots, U_{n}\right\}$ arranged in the increasing order).

Lemma 1. Let $U$ be a random variable with uniform distribution in $[0,1]$. Then the random variable $X=\varphi^{\leftarrow}(U)$ has the distribution function $F$.

The proof is straightforward:

$$
\mathbf{P}\left\{\varphi^{\leftarrow}(U) \leqslant x\right\}=\mathbf{P}\{U \geqslant \varphi(x)\}=1-\varphi(x)=F(x),
$$

where we have used the property (22).

Thanks to this lemma, we may assume without loss of generality that the given sequence $\left(X_{n}, n \geqslant 1\right)$ is represented as $\left(\varphi^{\leftarrow}\left(U_{n}\right), n \geqslant 1\right)$, where $U_{1}, U_{2}, \ldots$ is an auxiliary sequence of independent uniform random variables. In particular, it follows that the joint distribution of $\varphi^{\leftarrow}\left(U_{1, n}\right), \ldots, \varphi^{\leftarrow}\left(U_{n, n}\right)$ coincides with that of the original order statistics $X_{1, n}, \ldots, X_{n, n}$.

In turn, it is often handy to realize the uniform order statistics as the consecutive ratios of the sums of exponential random variables (see, e.g., [7, section III.3]).

Lemma 2. The joint distribution of the uniform order statistics $U_{1, n}, \ldots, U_{n, n}$ coincides with that of the random variables

$$
\frac{T_{1}}{T_{n+1}}, \ldots, \frac{T_{n}}{T_{n+1}},
$$

where $T_{i}=Y_{1}+\cdots+Y_{i}(i=1, \ldots, n+1)$ and $Y_{1}, \ldots, Y_{n+1}$ are independent random variables with common exponential distribution (with parameter 1 ):

$$
\mathbf{P}\{Y>x\}=e^{-x}, \quad x>0 .
$$

The following lemma (see, e.g., [15, section 0.4, Proposition 0.8 (v)]) guarantees that passing from $X_{i}$ to $U_{i}$ preserves the property of regular variation.

Lemma 3. Suppose that the function $\varphi(x)=1-F(x)$ is regularly varying at infinity with the exponent $0 \leqslant \alpha \leqslant \infty$, that is, condition (7) holds. Then the inverse function $\varphi^{\leftarrow}$ defined by (21) is regularly varying at zero with the exponent $1 / \alpha$, that is, it satisfies the condition

$$
\lim _{u \rightarrow+0} \frac{\varphi^{\leftarrow}(t u)}{\varphi^{\leftarrow}(u)}=t^{-1 / \alpha}, \quad \forall t>0 .
$$


The following basic formula, known as the Karamata representation, describes the general form of regularly varying functions (see, e.g., [7, section VIII.9] or [15, section 0.4, Corollary to Theorem 0.6]). We shall cite this result in the form convenient for our purposes.

Lemma 4. The function $\varphi^{\leftarrow}$ is regularly varying at zero (with exponent $1 / \alpha)$ if and only if it can be represented in the form

$$
\varphi^{\leftarrow}(u)=u^{-1 / \alpha} c(u) \exp \left(\int_{u}^{1} v^{-1} \gamma(v) d v\right)
$$

where $\lim _{u \rightarrow+0} c(u)=c(0<c<\infty), \lim _{u \rightarrow+0} \gamma(u)=0$.

Actually, we will need the following consequence from the Karamata representation, which is a modification of a lemma in [7, section VIII.8] to the case of the ratio of two values of the function (cf. [15, section 0.4 , Proposition 0.8 (ii)]).

Lemma 5. Under the conditions of Lemma 4, for any $\lambda>0$ there exists such $\delta>0$ that for any $0<u_{1}<u_{2}<\delta$ the following inequalities hold:

$$
\frac{1}{1+\lambda}\left(\frac{u_{1}}{u_{2}}\right)^{1 / \alpha+\lambda} \leqslant \frac{\varphi^{\leftarrow}\left(u_{2}\right)}{\varphi^{\leftarrow}\left(u_{1}\right)} \leqslant(1+\lambda)\left(\frac{u_{1}}{u_{2}}\right)^{1 / \alpha-\lambda}
$$

$\mathrm{P}$ r o of. Given $\lambda>0$, by Lemma 4 we can find such $\delta>0$ that, whenever $0<u<\delta$,

$$
\frac{2}{2+\lambda}=1-\frac{\lambda}{2+\lambda} \leqslant \frac{c(u)}{c} \leqslant 1+\frac{\lambda}{2+\lambda}=\frac{2(1+\lambda)}{2+\lambda}
$$

and

$$
-\lambda \leqslant \gamma(u) \leqslant \lambda .
$$

Substituting these inequalities into (23), we obtain

$$
\begin{aligned}
& \frac{1}{1+\lambda}\left(\frac{u_{1}}{u_{2}}\right)^{1 / \alpha} \exp \left(-\lambda \int_{u_{1}}^{u_{2}} \frac{d v}{v}\right) \leqslant \frac{\varphi^{\leftarrow}\left(u_{2}\right)}{\varphi^{\leftarrow}\left(u_{1}\right)} \\
& \leqslant(1+\lambda)\left(\frac{u_{1}}{u_{2}}\right)^{1 / \alpha} \exp \left(\lambda \int_{u_{1}}^{u_{2}} \frac{d v}{v}\right)
\end{aligned}
$$

which, on evaluation of the integral, yields (24).

We will also require the following general fact about the asymptotic behaviour of the maximal order statistic (see [8, section 4.3, Corollary 4.3.1]).

Lemma 6. Let $\left(X_{n}, n \geqslant 1\right)$ be independent identically distributed random variables (not necessarily positive), $X_{1, n}=\max _{1 \leqslant i \leqslant n} X_{i}$, and $\left(r_{n}\right.$, $n \geqslant 1)$ a nondecreasing sequence of numbers. Then

$$
\mathbf{P}\left\{X_{1, n} \geqslant r_{n} \text { i.o. }\right\}=0 \text { or } 1
$$

4 Теория вероятностей и ее применения, № 4 
according as

$$
\sum_{n=1}^{\infty} \mathbf{P}\left\{X_{n} \geqslant r_{n}\right\}<\infty \quad \text { or }=\infty .
$$

R e m a r k 5. We will only need the first part of this lemma.

\section{Proofs of the theorems.}

$\mathrm{P}$ r o of of $\mathrm{T} \mathrm{h}$ e o r e m 1. According to (6), the greatest atom $\mu_{1, n}$ is represented as

$$
\mu_{1, n}=\frac{X_{1, n}}{S_{n}}=\frac{X_{1, n} n^{-1}}{S_{n} n^{-1}} .
$$

By the strong law of large numbers, the denominator on the right-hand side of (25) a.s. tends to the expectation $0<\mathbf{E}\left[X_{1}\right]<\infty$ as $n \rightarrow \infty$. Let us show that $X_{1, n} n^{-1} \rightarrow 0$ with probability 1 . For this, it suffices to verify that for each $\varepsilon>0$

$$
\mathbf{P}\left\{X_{1, n}>n \varepsilon \text { i.o. }\right\}=0 \text {. }
$$

By Lemma 6, the condition (26) holds if and only if

$$
\sum_{n=1}^{\infty} \mathbf{P}\left\{X_{1}>n \varepsilon\right\}<\infty .
$$

In our case, the convergence of the series (27) follows from the finiteness of the expectation $\mathbf{E}\left[X_{1}\right]$. Indeed, integrating by parts we have

$$
\begin{aligned}
\mathbf{E}\left[X_{1}\right] & =\int_{0}^{\infty} x d F(x)=-\int_{0}^{\infty} x d \varphi(x)=\int_{0}^{\infty} \varphi(x) d x \\
& =\sum_{n=1}^{\infty} \int_{(n-1) \varepsilon}^{n \varepsilon} \varphi(x) d x \geqslant \sum_{n=1}^{\infty} \varepsilon \varphi(n \varepsilon)=\varepsilon \sum_{n=1}^{\infty} \mathbf{P}\left\{X_{1}>n \varepsilon\right\} .
\end{aligned}
$$

Note that in the outcome of the integration by parts in (28), the out-ofintegral term vanishes thanks to $\mathrm{E}\left[X_{1}\right]<\infty$ :

$$
b \varphi(b) \leqslant \int_{b}^{\infty} x d \varphi(x)=o(1) \quad \text { as } b \rightarrow+\infty \text {. }
$$

Thus, $\mu_{1, n} \rightarrow 0$ a.s. and we are done.

$\mathrm{P}$ r o of of $\mathrm{Th}$ e o r e m 2. Part (i) is an immediate corollary to a general result by Kesten [12] stating that, with probability 1,

$$
\limsup _{n \rightarrow \infty} \frac{\left|X_{n}\right|}{\left|S_{n-1}\right|}=\infty
$$

whenever $\left(X_{n}\right)_{n \geqslant 1}$ (not necessarily positive) are independent and identically distributed with $\mathbf{E}\left[\left|X_{1}\right|\right]=\infty$. Indeed, for positive summands this statement amounts to

$$
\limsup _{n \rightarrow \infty} \frac{X_{n}}{S_{n}}=1
$$


whence (8) obviously follows, with the role of $X_{1, n}$ played by the $X_{n}$.

For the proof of part (ii), we shall require a lemma on the a.s-behaviour of the uniform order statistics $U_{1, n}<\cdots<U_{n, n}$ (with probability 1 , all the inequalities are strict). Let us partition the interval $(0,1]$ by the points $t_{i}=i / n$ into the «boxes» $\Delta_{i, n}=\left(t_{i-1}, t_{i}\right], i=1, \ldots, n$, and set

$$
\nu_{i, n}:=\sum_{j=1}^{n} I\left\{U_{j} \in \Delta_{i, n}\right\}, \quad i=1, \ldots, n
$$

(here and below $I(A)$ stands for the indicator of event $A$ ). That is to say, $\nu_{i, n}$ is the number of random points $U_{1}, \ldots, U_{n}$ falling into the box $\Delta_{i, n}$. In the following lemma we shall use the notation $\log _{(2)}:=\log \log , \log _{(3)}:=$ $\log \log \log$, etc., where logarithms are taken to base 2 .

Lemma 7. Define the events

$$
\begin{aligned}
& A_{n}:=\left\{\nu_{1, n}=0\right\}=\left\{U_{1, n}>n^{-1}\right\}, \\
& B_{n}:=\left\{\nu_{2, n} \geqslant r_{n}\right\},
\end{aligned}
$$

where

$$
r_{n}:=\left[\frac{\log _{(3)} n}{\log _{(4)} n}\right]
$$

$[\cdot]$ denoting the «integer part». Then

$$
\mathbf{P}\left(A_{n} \cap B_{n} \text { i.o. }\right)=1 \text {. }
$$

P r o of of $\mathrm{L}$ e $\mathrm{m} \mathrm{ma} 7$. Wishing to apply the Borel-Cantelli lemma, we need to pass from the events $A_{n} \cap B_{n}$ to suitably constructed independent events. Consider the subsequence $n_{k}=2^{2^{k}}$; obviously, it suffices to prove the statement of the lemma for such $n$. Observe that

$$
\mathbf{P}\left\{U_{1, n_{k-1}} \leqslant n_{k}^{-1} \text { i.o. }\right\}=0 .
$$

Indeed, note that $n_{k}=n_{k-1}^{2}$ and that

$$
\mathbf{P}\left\{U_{1, n} \leqslant n^{-2} \text { i.o. }\right\}=0 .
$$

The latter follows from Lemma 6 as applied to random variables $1 / U_{i}$, because

$$
\sum_{n} \mathbf{P}\left\{\frac{1}{U_{1} \geqslant n^{2}}\right\}=\sum_{n} \mathbf{P}\left\{U_{1} \leqslant \frac{1}{n^{2}}\right\}=\sum_{n} n^{-2}<\infty .
$$

On the other hand, in view of (29)

$$
\nu_{2, n_{k}} \geqslant \widetilde{\nu}_{2, n_{k}}:=\sum_{j=n_{k-1}+1}^{n_{k}} I\left\{U_{j} \in \Delta_{2, n_{k}}\right\} .
$$


By combining (33) and (34), it is seen that our lemma will be proved once we show that

$$
\mathbf{P}\left(\widetilde{A}_{k} \cap \widetilde{B}_{k} \text { i.o. }\right)=1
$$

with $\widetilde{A}_{k}, \widetilde{B}_{k}$ defined as

$$
\begin{aligned}
& \widetilde{A}_{k}:=\left\{\min _{n_{k-1}+1 \leqslant j \leqslant n_{k}} U_{j}>n_{k}^{-1}\right\}, \\
& \widetilde{B}_{k}:=\left\{\widetilde{\nu}_{2, n_{k}} \geqslant r_{n_{k}}\right\}
\end{aligned}
$$

(cf. (30), (31)). But the events $\widetilde{A}_{k} \cap \widetilde{B}_{k}$ belong to the $\sigma$-algebras generated by the random variables $\left\{U_{j}, n_{k-1}+1 \leqslant j \leqslant n_{k}\right\}$, respectively, and hence they are independent, as desired. By the Borel-Cantelli lemma, it remains to verify that the series $\sum_{k} \mathbf{P}\left(\widetilde{A}_{k} \cap \widetilde{B}_{k}\right)$ is divergent.

Let us notice that, given the condition $\widetilde{A}_{k}$, the conditional distribution of each random variable $U_{j}, n_{k-1}+1 \leqslant j \leqslant n_{k}$, is uniform in the interval $\left(n_{k}^{-1}, 1\right]$. Indeed, for $n_{k}^{-1}<x \leqslant 1$ we have

$$
\mathbf{P}\left\{U_{j} \leqslant x \mid \tilde{A}_{k}\right\}=\mathbf{P}\left\{U_{j} \leqslant x \mid U_{j}>n_{k}^{-1}\right\}=\frac{\mathbf{P}\left\{n_{k}^{-1}<U_{j} \leqslant x\right\}}{\mathbf{P}\left\{U_{j}>n_{k}^{-1}\right\}}=\frac{x-n_{k}^{-1}}{1-n_{k}^{-1}} .
$$

Similar computation shows that these random variables are conditionally independent.

Therefore,

$$
\begin{aligned}
\mathbf{P}\left(\tilde{A}_{k} \cap \widetilde{B}_{k}\right) & =\mathbf{P}\left(\widetilde{A}_{k}\right) \cdot \mathbf{P}\left(\widetilde{B}_{k} \mid \widetilde{A}_{k}\right) \\
& =\left(1-\frac{1}{n_{k}}\right)^{\widetilde{n}_{k}} \sum_{i=r_{n_{k}}}^{\widetilde{n}_{k}}\left(\begin{array}{c}
\widetilde{n}_{k} \\
i
\end{array}\right) p_{k}^{i}\left(1-p_{k}\right)^{\widetilde{n}_{k}-i}
\end{aligned}
$$

where

$$
\begin{aligned}
& \tilde{n}_{k}:=n_{k}-n_{k-1}=n_{k}-\sqrt{n_{k}}, \\
& p_{k}:=\mathbf{P}\left\{n_{k}^{-1}<U_{1} \leqslant 2 n_{k}^{-1} \mid U_{1}>n_{k}^{-1}\right\}=\frac{n_{k}^{-1}}{1-n_{k}^{-1}}=\frac{1}{n_{k}-1},
\end{aligned}
$$

and $\left(\begin{array}{c}n \\ i\end{array}\right)=n ! /(i !(n-i) !)$ are the binomial coefficients.

To make the formulas less cumbersome, let us for a moment omit the subindices and write $n, \widetilde{n}, p$ and $r$ in place of $n_{k}, \widetilde{n}_{k}, p_{k}$ and $r_{n_{k}}$, respectively. Hence, (36) and (37) are rewritten as $\tilde{n}=n-\sqrt{n}$ and $p=1 /(n-1)$. We also note from (32) that

$$
r=\left[\frac{\log _{(3)} n}{\log _{(4)} n}\right]=o(\sqrt{n})
$$


Then, using Stirling's formula, the right-hand side of (35) can be estimated from below by

$$
\begin{aligned}
\left(1-\frac{1}{n}\right)^{n}\left(\begin{array}{c}
\widetilde{n} \\
i
\end{array}\right) p^{r}(1-p)^{n} & \sim \text { const } \cdot \widetilde{n}^{\widetilde{n}+1 / 2} r^{-r-1 / 2}(\widetilde{n}-r)^{-\widetilde{n}+r-1 / 2} p^{r} \\
& \geqslant \text { const } \cdot r^{-r-1 / 2}(\widetilde{n} p)^{r} \sim \text { const } \cdot r^{-r-1 / 2} \\
& =\text { const } \cdot 2^{-(r+1 / 2) \log r} \\
& \geqslant \text { const } \cdot 2^{-\log _{(3)} n-1 / 2 \log _{(4)} n} .
\end{aligned}
$$

Recalling that $n=n_{k}=2^{2^{k}}$, the right-hand side of (38) amounts to

$$
\text { const } \cdot 2^{-\log k-(1 / 2) \log _{(2)} k}=\frac{\text { const }}{k \sqrt{\log k}},
$$

which is the term of a divergent series, as required.

It is now easy to complete the proof of part (ii). Let us take a (random) subsequence $n^{\prime \prime}$ of the numbers $n$ provided by Lemma 7 , for which, with probability $1, U_{1, n}>1 / n$ and $\nu_{2, n} \geqslant r_{n} \rightarrow \infty$. Using Lemma 1 , we can write

$$
\begin{aligned}
\mu_{1, n} & =\frac{X_{1, n}}{S_{n}}=\left(\sum_{j=1}^{n} \frac{X_{j, n}}{X_{1, n}}\right)^{-1}=\left(\sum_{j=1}^{n} \frac{\varphi^{\leftarrow}\left(U_{j, n}\right)}{\varphi^{\leftarrow}\left(U_{1, n}\right)}\right)^{-1} \\
& \leqslant\left(\sum_{j=1}^{\nu_{2, n}} \frac{\varphi^{\leftarrow}\left(U_{j, n}\right)}{\varphi^{\leftarrow}\left(U_{1, n}\right)}\right)^{-1} .
\end{aligned}
$$

Note that

$$
U_{j, n} \leqslant \frac{2}{n} \leqslant 2 U_{1, n},
$$

because both $U_{1, n}$ and $U_{j, n}$ are contained in $\Delta_{2}$. As the function $\varphi^{\leftarrow}$ is nonincreasing, we can insert the inequality (40) into (39) to obtain

$$
\mu_{1, n} \leqslant \frac{1}{\nu_{2, n}} \cdot \frac{\varphi^{\leftarrow}\left(U_{1, n}\right)}{\varphi^{\leftarrow}\left(2 U_{1, n}\right)} .
$$

Finally, since $U_{1, n} \rightarrow 0$ a.s., Lemma 3 applies to yield

$$
\mu_{1, n} \leqslant \frac{1}{\nu_{2, n}} \cdot \frac{\varphi^{\leftarrow}\left(U_{1, n}\right)}{\varphi^{\leftarrow}\left(2 U_{1, n}\right)} \sim \frac{2^{1 / \alpha}}{\nu_{2, n}} \rightarrow 0,
$$

and the theorem is proved.

$\mathrm{P}$ r o of of $\mathrm{Th}$ e o r e m 3. We have to verify that the expectation of the «tail»

$$
0 \leqslant 1-\sigma_{k, n}=\sum_{i>k} \mu_{i, n}=\frac{1}{S_{n}} \sum_{i>k} X_{i, n}
$$


vanishes as $n$ and, afterwards, $k$ go to infinity. Consider the truncated random variables

$$
X_{i}^{(b)}=X_{i} \cdot I\left\{X_{i} \leqslant b\right\},
$$

where $b>0$ will be specified later, and split the set of indices $\{i: k<i \leqslant n\}$ into two parts:

$$
\begin{aligned}
& \mathscr{I}_{1}:=\left\{i>k: X_{i, n} \leqslant b\right\} \\
& \mathscr{I}_{2}:=\left\{i>k: X_{i, n}>b\right\}
\end{aligned}
$$

Note that

$$
\mathbf{P}\left\{X_{1, n} \leqslant b \text { i.o. }\right\}=0,
$$

because $X_{1, n}=\varphi^{\leftarrow}\left(U_{1, n}\right) \rightarrow+\infty$ a.s. Therefore, without loss of generality one may assume that $1 \in \mathscr{I}_{2}$.

Correspondingly, we can write

$$
\begin{aligned}
\sum_{i>k} \mu_{i, n} & =\sum_{i \in \mathscr{I}_{1}} \mu_{i, n}+\sum_{i \in \mathscr{I}_{2}} \mu_{i, n} \\
& \leqslant \frac{1}{S_{n}} \sum_{i=1}^{n} X_{i}^{(b)}+\sum_{i \in \mathscr{I}_{2}} \frac{X_{i, n}}{X_{1, n}}=\frac{S_{n}^{(b)} n^{-1}}{S_{n} n^{-1}}+\sum_{i \in \mathscr{I}_{2}} \frac{\varphi^{\leftarrow}\left(U_{i, n}\right)}{\varphi^{\leftarrow}\left(U_{1, n}\right)},
\end{aligned}
$$

where

$$
S_{n}^{(b)}=\sum_{i=1}^{n} X_{i}^{(b)} .
$$

We remark that for large $n$, the first term on the right-hand side of (44) is small with probability 1 (and also in mean, as it is bounded). Indeed, since the expectation of $X_{i}^{(b)}$ is finite, by the strong law of large numbers with probability 1

$$
\frac{S_{n}^{(b)}}{n} \rightarrow \mathbf{E}\left[X_{1}^{(b)}\right]<\infty
$$

whereas $S_{n} / n$, thanks to positivity of the summands, a.s. goes to infinity:

$$
\liminf _{n \rightarrow \infty} \frac{S_{n}}{n} \geqslant \lim _{n \rightarrow \infty} \frac{S_{n}^{(b)}}{n}=\mathbf{E}\left[X_{1}^{(b)}\right] \uparrow+\infty \quad \text { as } \quad b \uparrow+\infty .
$$

Turning to the second term in (44) where $U_{i, n}$ are supposed to be small, we wish to make use of regular variation of the function $\varphi^{\leftarrow}$ (see Lemma 3) and, roughly speaking, to replace the ratio $\varphi^{\leftarrow}\left(U_{i, n}\right) / \varphi^{\leftarrow}\left(U_{1, n}\right)$ by $\left(U_{i, n} / U_{1, n}\right)^{-1 / \alpha}$. More precisely, pick a $\lambda>0$ such that $\lambda<1 / \alpha-1$ and define the number $\rho$ as

$$
\rho:=\frac{1}{\alpha}-\lambda>1
$$


Then, by Lemma 5 , there exists a $\delta>0$ such that for $0<u_{1}<u_{2}<\delta$

$$
\frac{\varphi^{\leftarrow}\left(u_{2}\right)}{\varphi^{\leftarrow}\left(u_{1}\right)} \leqslant(1+\lambda)\left(\frac{u_{1}}{u_{2}}\right)^{1 / \alpha-\lambda}=(1+\lambda)\left(\frac{u_{1}}{u_{2}}\right)^{\rho} \text {. }
$$

Finally, let us put

$$
b:=\varphi^{\leftarrow}(\delta)
$$

thus choosing the truncation parameter used in (41). Then for each $i \in \mathscr{I}_{2}$ we have that $0<U_{i, n}<\delta$. Indeed, assume to the contrary that $U_{i, n} \geqslant \delta$, then by monotonicity of the function $\varphi^{\leftarrow}$ we would get

$$
X_{i, n}=\varphi^{\leftarrow}\left(U_{i, n}\right) \leqslant \varphi^{\leftarrow}(\delta)=b,
$$

which contradicts the definition (43) of the set $\mathscr{I}_{2}$. So, we may apply the inequality (46) to the pair $U_{1, n}, U_{i, n}$ to obtain

$$
\begin{aligned}
\mathbf{E}\left[\sum_{i \in \mathscr{I}_{2}} \frac{\varphi^{\leftarrow}\left(U_{i, n}\right)}{\varphi^{\leftarrow}\left(U_{1, n}\right)}\right] & \leqslant \mathbf{E}\left[\sum_{i \in \mathscr{I}_{2}}(1+\lambda)\left(\frac{U_{1, n}}{U_{i, n}}\right)^{\rho}\right] \\
& \leqslant(1+\lambda) \sum_{i=k+1}^{n} \mathbf{E}\left[\left(\frac{U_{1, n}}{U_{i, n}}\right)^{\rho}\right] .
\end{aligned}
$$

By Lemma 2, we can rewrite the right-hand side of (48) (omitting the constant factor) as

$$
\begin{aligned}
\sum_{i=k+1}^{n} \mathbf{E}\left[\left(\frac{T_{1}}{T_{i}}\right)^{\rho}\right] & \leqslant \sum_{i=k+1}^{n} \mathbf{E}\left[T_{1}^{\rho} \cdot\left(T_{i}-T_{1}\right)^{-\rho}\right] \\
& \leqslant \sum_{i=k+1}^{n} \mathbf{E}\left[T_{1}^{\rho}\right] \cdot \mathbf{E}\left[\left(T_{i}-T_{1}\right)^{-\rho}\right]
\end{aligned}
$$

because the random variables $T_{1}=Y_{1}$ and $T_{i}-T_{1}=Y_{2}+\cdots+Y_{i}$ are independent of each other. Using that the random variables $T_{i}$ have the gamma distribution (see, e.g., [7, section III.3]) with the density

$$
f_{i}(x)=\frac{1}{\Gamma(i)} x^{i-1} e^{-x}, \quad x>0
$$

the expectations on the right-hand side of (49) can be easily computed:

$$
\begin{aligned}
\mathbf{E}\left[T_{1}^{\rho}\right] & =\int_{0}^{\infty} x^{\rho} f_{1}(x) d x=\int_{0}^{\infty} x^{\rho} e^{-x} d x=\Gamma(\rho+1), \\
\check{\mathbf{E}}\left[\left(T_{i}-T_{1}\right)^{-\rho}\right] & =\int_{0}^{\infty} x^{-\rho} f_{i-1}(x) d x=\frac{1}{\Gamma(i-1)} \int_{0}^{\infty} x^{i-\rho-2} e^{-x} d x \\
& =\frac{\Gamma(i-\rho-1)}{\Gamma(i-1)}
\end{aligned}
$$


(we assume that $i>\rho+1$ ). Substituting these expressions into (49) and returning to the estimate (48), we obtain

$$
\mathbf{E}\left[\sum_{i \in \mathscr{I}_{2}} \mu_{i, n}\right] \leqslant \text { const } \cdot \sum_{i=k+1}^{\infty} \mathbf{E}\left[\frac{\Gamma(i-\rho-1)}{\Gamma(i-1)}\right] .
$$

By means of Stirling's formula for the gamma function

$$
\Gamma(s+1) \sim \sqrt{2 \pi} s^{s+\frac{1}{2}} e^{-s} \text { as } s \rightarrow \infty,
$$

it is easy to check that

$$
\frac{\Gamma(i-\rho-1)}{\Gamma(i-1)} \sim i^{-\rho} \text { as } i \rightarrow \infty,
$$

and therefore the series in (50) is convergent (recall that by the condition (45), $\rho>1)$. Hence, for $k$ large enough the right-hand side of (50) becomes arbitrarily small, which completes the proof.

$\mathrm{Pr}$ o of of $\mathrm{T} \mathrm{h}$ e or e $\mathrm{m} \mathrm{4}$. This follows immediately from the proof of Theorem 3 since the right-hand side of the estimate (50) is independent of $n$.

P r o of of $\mathrm{Th}$ e or e m 5. We follow the strategy of the proof of Theorem 3. Given $\varepsilon>0$, consider the probability

$$
\mathbf{P}\left\{\mu_{1, n}>\varepsilon\right\}=\mathbf{P}\left\{\frac{X_{1, n}}{S_{n}}>\varepsilon\right\}=\mathbf{P}\left\{\sum_{i=1}^{n} \frac{X_{i, n}}{X_{1, n}}<\frac{1}{\varepsilon}\right\} .
$$

Let us fix an arbitrary number $\lambda>0$. Applying Lemma 5 (with $\alpha=1$ ), we find a $\delta>0$ such that for any $0<u_{1}<u_{2}<\delta$

$$
\frac{\varphi^{\leftarrow}\left(u_{2}\right)}{\varphi^{\leftarrow}\left(u_{1}\right)} \geqslant \frac{1}{1+\lambda}\left(\frac{u_{1}}{u_{2}}\right)^{1+\lambda}
$$

Define the index sets

$$
\mathscr{I}_{1}:=\left\{i: U_{i, n} \geqslant \delta\right\}, \quad \mathscr{I}_{2}:=\left\{i: U_{i, n}<\delta\right\} .
$$

Since $U_{1, n} \rightarrow 0$ with probability 1 , we may and will assume that $U_{1, n}<\delta$, that is, $1 \in \mathscr{I}_{2}$.

Retaining in the sum on the right-hand side of (51) the summands with $i \in \mathscr{I}_{2}$ only and applying the inequality (52) to the pair $U_{1, n}, U_{i, n}$, we get

$$
\limsup _{n \rightarrow \infty} \mathbf{P}\left\{\mu_{1, n}>\varepsilon\right\} \leqslant \limsup _{n \rightarrow \infty} \mathbf{P}\left\{\sum_{i \in \mathscr{I}_{2}}\left(\frac{U_{1, n}}{U_{i, n}}\right)^{1+\lambda}<\frac{1+\lambda}{\varepsilon}\right\} .
$$


Let us show that we may pass back in the present result to the complete sum, that is, over all $i=1, \ldots, n$. Set

$$
W_{i}:=\frac{1}{U_{i}} \cdot I\left\{U_{i} \geqslant \delta\right\}, \quad i=1, \ldots, n .
$$

Then, as $n \rightarrow \infty$, we have that

$$
\sum_{i \in \mathscr{I}_{1}}\left(\frac{U_{1, n}}{U_{i, n}}\right)^{1+\lambda}=n U_{1, n}^{1+\lambda} \cdot \frac{1}{n} \sum_{i=1}^{n} W_{i}^{1+\lambda} \rightarrow 0
$$

in probability. Indeed, by the law of large numbers

$$
\frac{1}{n} \sum_{i=1}^{n} W_{i}^{1+\lambda} \rightarrow \mathbf{E}\left[W_{1}^{1+\lambda}\right]<\infty
$$

whereas $n U_{1, n}^{1+\lambda} \rightarrow 0$, as can be easily verified by representing $U_{1, n}$ as $T_{1} / T_{n+1}$ (Lemma 2) and applying the law of large numbers:

$$
n\left(\frac{T_{1}}{T_{n+1}}\right)^{1+\lambda}=\frac{T_{1}^{1+\lambda}}{n^{\lambda}} \cdot\left(\frac{T_{n+1}}{n}\right)^{-1-\lambda} \rightarrow 0 .
$$

Hence, increasing the constant in the right-hand side inequality in (53), we can rewrite (53) as

$$
\begin{aligned}
\limsup _{n \rightarrow \infty} \mathbf{P}\left\{\mu_{1, n}>\varepsilon\right\} & \leqslant \limsup _{n \rightarrow \infty} \mathbf{P}\left\{\sum_{i=1}^{n}\left(\frac{U_{1, n}}{U_{i, n}}\right)^{1+\lambda}<\frac{1+2 \lambda}{\varepsilon}\right\} \\
& =\limsup _{n \rightarrow \infty} \mathbf{P}\left\{\sum_{i=1}^{n}\left(\frac{T_{1}}{T_{i}}\right)^{1+\lambda}<\frac{1+2 \lambda}{\varepsilon}\right\},
\end{aligned}
$$

where we once more used Lemma 2. Let us now notice that the series

$$
\sum_{n=1}^{\infty}\left(\frac{1}{T_{n}}\right)^{1+\lambda}
$$

is a.s.-convergent because, by the strong law of large numbers,

$$
\frac{T_{n}}{n}=\frac{Y_{1}+\cdots+Y_{n}}{n} \rightarrow \mathrm{E}\left[Y_{1}\right]=\int_{0}^{\infty} x e^{-x} d x=1 .
$$

Hence, the estimate (54) can be rewritten as

$$
\limsup _{n \rightarrow \infty} \mathbf{P}\left\{\mu_{1, n}>\varepsilon\right\} \leqslant \mathbf{P}\left\{Y_{1}^{1+\lambda} \sum_{n=1}^{\infty}\left(\frac{1}{T_{n}}\right)^{1+\lambda} \leqslant \frac{1+2 \lambda}{\varepsilon}\right\} .
$$


Moreover, (55) implies that, with probability 1, there exists such (random) number $n_{0}$ that $T_{n} \leqslant 2 n$ for all $n \geqslant n_{0}$. It then follows that the probability on the right-hand side of $(56)$ is no greater than

$$
\begin{aligned}
& \mathbf{P}\left\{Y_{1}^{1+\lambda} \sum_{n=n_{0}}^{\infty}\left(\frac{1}{T_{n}}\right)^{1+\lambda} \leqslant \frac{1+2 \lambda}{\varepsilon}\right\} \\
& \quad \leqslant \mathbf{P}\left\{Y_{1}^{1+\lambda} \sum_{n=n_{0}}^{\infty}\left(\frac{1}{2 n}\right)^{1+\lambda} \leqslant \frac{1+2 \lambda}{\varepsilon}\right\} .
\end{aligned}
$$

It is easy to see that

$$
\sum_{n=n_{0}}^{\infty} \frac{1}{n^{1+\lambda}} \geqslant \int_{n_{0}}^{\infty} \frac{1}{x^{1+\lambda}} d x=\frac{1}{\lambda n_{0}^{\lambda}} .
$$

Therefore, from (57) and (58) we finally get

$$
\limsup _{n \rightarrow \infty} \mathbf{P}\left\{\mu_{1, n}>\varepsilon\right\} \leqslant \mathbf{P}\left\{Y_{1}^{1+\lambda} \leqslant(1+2 \lambda) \varepsilon^{-1} \lambda n_{0}^{\lambda} 2^{1+\lambda}\right\} .
$$

Recall that $\lambda>0$ is arbitrary and note that $n_{0}$, by its choice, does not depend on $\lambda$. Passing to the limit in (59) as $\lambda \rightarrow+0$, we obtain

$$
\limsup _{n \rightarrow \infty} \mathbf{P}\left\{\mu_{1, n}>\varepsilon\right\} \leqslant \mathbf{P}\left\{Y_{1} \leqslant 0\right\}=0
$$

as required.

$\mathrm{Pr}$ o of of $\mathrm{Th}$ e o r e m 6. For $p$ being an integer, the statements (10) and (11) are contained in [5] $(p=1)$ and [2] $(p \geqslant 1)$. Extension to arbitrary $p>0$ is straightforward, e.g. by Lyapunov's inequality. It remains to show that (11) implies weak localization. Indeed, from (10) with $p=1$ we have $\lim _{n \rightarrow \infty} \mathbf{E}\left[\mu_{1, n}\right]=1$, whence in view of the inequalities $\mu_{1, n} \leqslant \sigma_{k, n} \leqslant 1$, the condition (L2) follows.

$\mathrm{Pr}$ o of of $\mathrm{T} \mathrm{h}$ e o r e m 7 . The statements (14)-(16) and (20) are adapted from [14, Theorems 2, 3]). The statement (17) follows from Theorem 2 (i) which can be applied because for $k^{*}<\infty$ one has $\mathbf{E}\left[X_{1}\right]=\infty$. Indeed, if $\mathbf{E}\left[X_{1}\right]<\infty$, then by Theorem $1, \lim _{n \rightarrow \infty} \mu_{1, n}=0$ a.s., which contradicts (16).

To check (18), note that

$$
k^{*} \mu_{k^{*}, n} \leqslant \sigma_{k^{*}, n} \leqslant\left(k^{*}-1\right) \mu_{1, n}+\mu_{k^{*}, n},
$$

whence

$$
\sigma_{k^{*}, n}-\left(k^{*}-1\right) \mu_{1, n} \leqslant \mu_{k^{*}, n} \leqslant \frac{1}{k^{*}} \sigma_{k^{*}, n} .
$$

Combined with (15) this yields

$$
\limsup _{n \rightarrow \infty} \mu_{k^{*}, n} \leqslant \frac{1}{k^{*}} .
$$


On the other hand, by (16), for any $\varepsilon>0$ with probability 1 we have that $\mu_{1, n}<1 / k^{*}+\varepsilon$ i.o. and hence from (60)

$$
\mu_{k^{*}, n} \geqslant \sigma_{k^{*}, n}-1+\frac{1}{k^{*}}-\left(k^{*}-1\right) \varepsilon \text { i.o. }
$$

Keeping in mind (15), let $n \rightarrow \infty$ and $\varepsilon \rightarrow 0$ to obtain

$$
\limsup _{n \rightarrow \infty} \mu_{k^{*}, n} \geqslant \frac{1}{k^{*}} .
$$

As a result, the inequalities (61) and (62) yield (18).

Next, the statement (19) immediately follows from the identity $\sigma_{k^{*}-1, n}=\sigma_{k^{*}, n}-\mu_{k^{*}, n}$ and formulas (15), (18).

Finally, to show that (20) inhibits the condition (L4) of strong localization, it suffices to notice that $\sigma_{k, n} \leqslant k \mu_{1, n}$, and so (20) implies that $\liminf _{n \rightarrow \infty} \sigma_{k, n}=0$ with probability 1 .

6. Appendix.

$\mathrm{P}$ r o of of $\mathrm{P}$ r op osition 1 . We first show that (L1) $\Leftrightarrow(\mathrm{L} 2)$. Let us fix $k \geqslant 1$ and $\lambda>0$. Using (2), (5), and (3), we have

$$
\begin{aligned}
m_{\lambda}^{(n)} & \leqslant \sum_{i=1}^{k}\left(\mu_{i, n}\right)^{1+\lambda}+\left(\mu_{k, n}\right)^{\lambda} \cdot \sum_{i>k} \mu_{i, n} \\
& \leqslant \sum_{i=1}^{k} \mu_{i, n}+\left(\mu_{k, n}\right)^{\lambda}=\sigma_{k, n}+\left(\mu_{k, n}\right)^{\lambda} .
\end{aligned}
$$

Note that

$$
1=\sum_{i} \mu_{i, n} \geqslant \sum_{i=1}^{k} \mu_{i, n} \geqslant k \mu_{k, n},
$$

whence

$$
\mu_{k, n} \leqslant k^{-1} .
$$

Substituting (64) into (63) and taking the expectation yields

$$
\mathbf{E}\left[m_{\lambda}^{(n)}\right] \leqslant \mathbf{E}\left[\sigma_{k, n}\right]+k^{-\lambda} .
$$

Consecutively passing to the limit in (65) as $n \rightarrow \infty, k \rightarrow \infty$ and $\lambda \rightarrow+0$, we obtain:

$$
\begin{aligned}
& \liminf _{n \rightarrow \infty} \mathbf{E}\left[m_{\lambda}^{(n)}\right] \leqslant \liminf _{n \rightarrow \infty} \mathbf{E}\left[\sigma_{k, n}\right]+k^{-\lambda}, \\
& \liminf _{n \rightarrow \infty} \mathbf{E}\left[m_{\lambda}^{(n)}\right] \leqslant \lim _{k \rightarrow \infty} \liminf _{n \rightarrow \infty} \mathbf{E}\left[\sigma_{k, n}\right]
\end{aligned}
$$

and, finally,

$$
\lim _{\lambda \rightarrow+0} \liminf _{n \rightarrow \infty} \mathbf{E}\left[m_{\lambda}^{(n)}\right] \leqslant \lim _{k \rightarrow \infty} \liminf _{n \rightarrow \infty} \mathbf{E}\left[\sigma_{k, n}\right] .
$$


On the other hand, by Hölder's inequality

$$
\begin{aligned}
\sigma_{k, n} & =\sum_{i=1}^{k} \mu_{i, n} \leqslant k^{\lambda /(1+\lambda)} \cdot\left(\sum_{i=1}^{k}\left(\mu_{i, n}\right)^{1+\lambda}\right)^{1 /(1+\lambda)} \\
& \leqslant k^{\lambda /(1+\lambda)} \cdot\left(m_{\lambda}^{(n)}\right)^{1 /(1+\lambda)}
\end{aligned}
$$

so that

$$
m_{\lambda}^{(n)} \geqslant\left(\sigma_{k, n}\right)^{1+\lambda} k^{-\lambda}
$$

Let us take the expectation and again pass to the limit in (69) as $n \rightarrow \infty$, $\lambda \rightarrow+0$ and $k \rightarrow \infty$ (note that the order of limits is different from that used above in (66)-(68)):

$$
\begin{aligned}
\liminf _{n \rightarrow \infty} \mathbf{E}\left[m_{\lambda}^{(n)}\right] & \geqslant k^{-\lambda}\left(\liminf _{n \rightarrow \infty} \mathbf{E}\left[\sigma_{k, n}\right]\right)^{1+\lambda}, \\
\lim _{\lambda \rightarrow+0} \liminf _{n \rightarrow \infty} \mathbf{E}\left[m_{\lambda}^{(n)}\right] & \geqslant \liminf _{n \rightarrow \infty} \mathbf{E}\left[\sigma_{k, n}\right],
\end{aligned}
$$

and

$$
\lim _{\lambda \rightarrow+0} \liminf _{n \rightarrow \infty} \mathbf{E}\left[m_{\lambda}^{(n)}\right] \geqslant \lim _{k \rightarrow \infty} \liminf _{n \rightarrow \infty} \mathbf{E}\left[\sigma_{k, n}\right] .
$$

By combining the inequalities (68) and (71), we deduce that

$$
\lim _{\lambda \rightarrow+0} \liminf _{n \rightarrow \infty} \mathbf{E}\left[m_{\lambda}^{(n)}\right]=\lim _{k \rightarrow \infty} \liminf _{n \rightarrow \infty} \mathbf{E}\left[\sigma_{k, n}\right]
$$

and thus (L1) $\Leftrightarrow$ (L2).

Similarly as for the derivation of (72), one can show that

$$
\lim _{\lambda \rightarrow+0} \limsup _{n \rightarrow \infty} \mathbf{E}\left[m_{\lambda}^{(n)}\right]=\lim _{k \rightarrow \infty} \limsup _{n \rightarrow \infty} \mathbf{E}\left[\sigma_{k, n}\right]
$$

so that (D1) $\Leftrightarrow$ (D2).

Further, let us verify the equivalence of (L2) and (L3). Assume that (L3) holds. By Chebyshev's inequality,

$$
\mathbf{E}\left[\sigma_{k, n}\right] \geqslant(1-\varepsilon) \cdot \mathbf{P}\left\{\sigma_{k, n}>1-\varepsilon\right\},
$$

whence

$$
\lim _{k \rightarrow \infty} \liminf _{n \rightarrow \infty} \mathbf{E}\left[\sigma_{k, n}\right] \geqslant(1-\varepsilon) \lim _{k \rightarrow \infty} \liminf _{n \rightarrow \infty} \mathbf{P}\left\{\sigma_{k, n}>1-\varepsilon\right\}=1-\varepsilon .
$$

Since $\varepsilon>0$ is arbitrary and $\sigma_{k, n} \leqslant 1$, (L2) follows.

Conversely, let us be given (L2). Again applying Chebyshev's inequality (to the random variable $1-\sigma_{k, n}$ ), we have

$$
\mathbf{E}\left[1-\sigma_{k, n}\right] \geqslant \varepsilon \mathbf{P}\left\{1-\sigma_{k, n} \geqslant \varepsilon\right\},
$$


or

$$
\mathbf{E}\left[\sigma_{k, n}\right] \leqslant 1-\varepsilon+\varepsilon \mathbf{P}\left\{1-\sigma_{k, n} \geqslant \varepsilon\right\} .
$$

Passing here to the limit as $n \rightarrow \infty, k \rightarrow \infty$, and using the condition (L2) yields

$$
1 \leqslant 1-\varepsilon+\varepsilon \lim _{k \rightarrow \infty} \liminf _{n \rightarrow \infty} \mathbf{P}\left\{\sigma_{k, n}>1-\varepsilon\right\}
$$

whence (L3) follows.

The equivalence of (D2) and (D3) is established analogously, with the only modification that in the estimates (73) and (74) one should replace $\varepsilon$ by $1-\varepsilon$.

Finally, let us prove the implications (L4) $\Rightarrow$ (L2) and (D4) $\Rightarrow$ (D2). Using that $0 \leqslant \sigma_{k, n} \leqslant 1$ for all $k, n$, and twice applying the Fatou lemma (for $n \rightarrow \infty$ and $k \rightarrow \infty$ ), we get

$$
\begin{aligned}
1 & \geqslant \lim _{k \rightarrow \infty} \liminf _{n \rightarrow \infty} \mathbf{E}\left[\sigma_{k, n}\right] \geqslant \lim _{k \rightarrow \infty} \mathbf{E}\left[\liminf _{n \rightarrow \infty} \sigma_{k, n}\right] \\
& \geqslant \mathbf{E}\left[\lim _{k \rightarrow \infty} \liminf _{n \rightarrow \infty} \sigma_{k, n}\right] .
\end{aligned}
$$

Whenever (L4) is fulfilled, the right-hand side of (75) is equal to 1 , so that the inequalities (75) imply (L2). Similarly, using the Fatou lemma with lim sup in place of liminf, we obtain (D4) $\Rightarrow$ (D2).

Acknowledgements. The authors would like to thank S. A. Molchanov for fruitful discussions on the subject of the paper, and H. Rost, G. F. Dell'Antonio, K. A. Makarov, Ya. G. Sinai, and A. V. Bulinski for their stimulating interest and valuable comments made at various stages of this work. The second author gratefully acknowledges the kind hospitality lent at the Institute for Mathematics, Ruhr-University of Bochum, during his stay in the Fall of 1997.

The authors wish to dedicate this work to the memory of the prominent Russian probabilist Boris Vladimirovich Gnedenko (1912-1995). One can only guess how many works related to the theory of summation of independent random variables, if traced back through the bibliographic references, would eventually lead to the name of Gnedenko. Our paper also belongs to this (potentially infinite) set.

\section{REFERENCES}

1. Albeverio S., Molchanov S. A., Surgailis D. Stratified structure of the Universe and the Burgers equation: A probabilistic approach. - Probab. Theory Relat. Fields, 1994, v. 100, p. 457-484.

2. Аров Д. З., Бобров А. А. О крайних членах вариационного ряда и их роли в сумме независимых величин. - Теория вероятн. и ее примен., 1960, т. 5, № 4, c. $313-328$. 
3. Богачев Л. В. О локализации случайных дискретных мер. - Докл. АН СССР, 1997 , т. 357, c. $153-156$.

4. Bogachev L. V., Molchanov S. A. Mean-field models in the theory of random media. I. - Theor. Math. Phys., 1989, v. 81, p. 1207-1214.

5. Darling $D$. A. The influence of the maximal term in the addition of independent random variables. - Trans. Amer. Math. Soc., 1952, v. 73, p. 95-107.

6. Derrida $B$. Nonself-averaging effects in sums of random variables, spin glasses, random maps and random walks. - On Three Levels. Micro-, meso-, and macroapproaches in physics, M. Fannes, C. Maes, and A. Verbeure, eds., Proceedings of a NATO Adv. Res. Workshop, Leuven, Belgium, July 19-23, 1993. New York: Plenum Press, 1994, p. 125-137.

7. Феллер В. Введение в теорию вероятностей и ее приложения. Т. 2, М.: Мир, 1984, $752 \mathrm{c}$.

8. Галамбош Я. Асимптотическая теория экстремальных порядковых статистик. М.: Наука, 1984.

9. Гяеденко Б. В. Роль максимального слагаемого в суммах независимых случайных величин. - Укр. матем. журнал, 1953, т. 5, с. 291-298.

10. Гнедеяко Б. В., Колмогоров А. Н. Предельные распределения для сумм случайных независимых переменных. М.-Л.: Гостехиздат, 1949, 264 с.

11. Haeusler E., Mason D. M. On the asymptotic behavior of sums of order statistics from a distribution with a slowly varying upper tail. - Sums, Trimmed Sums and Extremes, M. G. Hahn, D. M. Mason, and D. C. Weiner, eds. Boston, Basel, Berlin: Birkhäuser, 1991, p. 355-376.

12. Kesten $H$. Sums of random variables with infinite expectation. - Amer. Math. Monthly, 1971, v. 78, p. 305-308.

13. Molchanov $S$. A. Ideas in the theory of random media. - Acta Appl. Math., 1991, v. 22, p. 139-282.

14. Pruitt $W . E$. The contribution to the sum of the summand of maximum modulus. Ann. Probab., 1987, v. 15, p. 885-896.

15. Resnick S. I. Extreme Values, Regular Variation, and Point Processes. New York, Berlin, Heidelberg: Springer-Verlag, 1987. 